two-dimensional data matrices for a pharmaceutical pedigree. He added that the pharmaceutical industry in Europe feels that RFID "is not sufficiently mature, is less robust and costs more" than other approaches. By itself, he said, RFID is not a panacea, but should be used where it fits best.

Despite the controversy, RFID technology has great potential. "In the context of healthcare, there are enormous gains to be had by increasing patient safety, and certifying the pedigree of pharmaceutical products as they move from the manufacturer to the final user," Cavoukian said. "RFID technology is not a negative, by any means. It all depends on its application."

\section{REFERENCES}

Cavoukian A (2006) Privacy Guidelines for RFID Information Systems. O ntario, Canada: Information and Privacy Commissioner. www.ipc.on.ca/docs/rfidgdlines.pdf

EGE (2005a) Ethical Aspects of ICT Implants in the Human Body. O pinion N o. 20, 16 M arch. Brussels, Belgium: European G roup on Ethics in Science and N ew Technologies

EGE (2005b) Ethical Aspects of ICT Implants in the Human Body: O pinion Presented to the Commission by the European G roup on Ethics. MEM O/05/97, 17 M arch. Brussels, Belgium: European Group on Ethics in Science and $\mathrm{N} \mathrm{ew}$ Technologies

FDA (2006) FDA Counterfeit D rug Task Force Report, 2006 U pdate. Rockville, MD, USA: Food and D rug Administration

\section{HowardWdinsky}

doi:10.1038/sj.embor.7400810

\title{
The costs of REACH
}

\author{
REACH islargely welcomed, but the requirement to test existing \\ chemicals for adverse effects is not good news for all
}

E arly next year, the European Union (EU ) plans to implement a major piece of legislation-about 1,200 pages in its current form-to replace 40 different laws regulating the use of chemicals across the member states. When the European Commission (EC) presented its first draft in M ay 2003 (EC, 2003), it immediately drew huge attention because of its far-reaching consequences, not only economic but also on human health and the environment. If passed, the REACH legislation will require the chemical industry to provide information on the potential negative effectsmost of which are still unknown- of a vast number of chemicals used throughout the EU . N ot surprisingly, this has met with criticism from all quarters. The costs of obtaining this information, and the subsequent effects on the European chemical industry, are hotly disputed.

REACH dictates new rules on the Registration, Evaluation and Authorisation of $\mathrm{CH}$ emicals. It requires manufacturers and downstream users of chemicals to register their substances-if they are sold in the EU as isolated compounds or in preparationseither with a national regulatory authority or a central European Chemical Agency, which the EC plans to establish next year in Helsinki, Finland. Companies are required to submit a registration dossier for each product, listing possible adverse effects on human health or the environment.

\section{[REACH] immediately drew huge attention because of its far- reaching consequences, not only economic but also on human health and the environment}

REACH also requires additional authorization for substances that the EC considers of "very high concern": chemicals that are carcinogenic, mutagenic or toxic to the reproductive system; endocrine disruptors; or persistent, bioaccumulative and toxic substances. Producers or downstream users must perform a risk assessment to ascertain whether their product creates risks to human health or the environment, which social benefits it provides, and whether safer alternatives are available. If, on the basis of this assessment, a regulatory agency reaches the conclusion that a product is hazardous and provides little or no benefits, the agency or the EC might ban it for specific or all uses, impose additional safety measures, or give the producer a deadline to substitute the product with a safer alternative. REACH therefore reverses the current procedurein which regulatory agencies must demonstrate that a specific chemical is hazardous before imposing a ban-and instead places the burden of proof on industry.

Furthermore, REACH will be the first law to require such information on all chemicals that are currently produced and used in the EU-about 100,000 substances overall. Although exceptions exist for synthetic intermediates, medications and substances that are produced in quantities of less than one tonne per year, REACH will still affect about 30,000 products. Less than one percent of these have been thoroughly tested for adverse effects to date, according to David Santillo, senior scientist at Greenpeace Research Laboratories, based at the University of Exeter, UK. For Edward Calabrese, Professor of Toxicology at the University of M assachusetts (Boston, USA), this is not surprising. "Society has not been able to [test all chemicals]. They have done that on the drug side but they haven't done that on the commercial chemicals side. It's kind of a mess," he said.

The first version of REACH was heralded by environmentalist and consumer protection groups, because it meant that industry was obliged to provide information on the safety of many chemicals in daily use. The European Parliament (EP) further strengthened the requirements for the risk assessment and expanded regulatory agencies' ability to ban substances with negative effects. However, the chemical industry, both within and outside Europe, maintain that the costs of providing extended safety data on all chemicals will hamper their international competitiveness. They have also criticized the procedure to ban or substitute a hazardous substance as being too strict. After intense lobbying from all sides, the EC presented its final proposal in $O$ ctober 2003. It now awaits ratification by the EP and the Council of Europe and, if both agree, will go into force in April 2007. It is not yet clear whether the text will undergo an additional round of negotiation; while the Council has taken a more industry-friendly position, the EP still calls for stricter requirements for authorization and substitution. 
T he debate over REACH continues. Environmental and consumer protection groups claim that the current proposal contains too many loopholes that would leave scores of hazardous substances on the market. Industry maintains that the final version does not address their main concerns. "There is the testing to start with," said Bennard van Ravenzwaay, Head of the Toxicology and Ecology U nit at the chemical producer BASF in Ludwigshafen, Germany. Industry will have to perform and pay for an extremely high number of additional tests.

These tests look at various effects, including toxicity, carcinogenicity, mutagenicity, reproductive toxicity and endocrine disrupting activities, and most must be conducted on animals. Ironically, the EC is also updating its directive on the use of animals in research with the overall aim of reducing the number of animals used (EEC, 1986; Matthiessen et al, 2003), a situation which van Ravenzwaay described as being caught "between hammer and anvil". Indeed, REACH is bad news for laboratory animals: the German Federal Institute for Risk Assessment (BfR; Berlin) estimated that the legislation could lead to a demand for up to 45 million laboratory animals over the next 15 years (H ofer et al, 2004).

Despite some alternatives, most toxicological tests still rely on animal studies, some of which can take years and use hundreds of animals. Demonstrating reproductive toxicity, for example, requires a twogeneration study to assess effects on the $F_{1}$ and $F_{2}$ generation. To complicate matters, the development of in vitro methods that would pass both scientific and regulatory scrutiny is a lengthy and costly undertaking. BASF spends $€ 2$ million each year developing in vitro alternatives but van Ravenzwaay is largely pessimistic that any new tests will be robust enough to be accepted by regulatory agencies when REACH goes into effect. Before being added to the EU Directive on Dangerous Substances (EEC, 1967), which now lists 92 accepted tests, any new in vitro method must first be validated by the European Centre for the Evaluation of Alternative Methods (Ispra, Italy) and approved by the EC and the $O$ rganization for Economic Cooperation and Development (Paris, France). Even new in vitro tests and advances in toxicogenomics cannot replace animal tests in the short term, believes Santillo. "It is promising but it is not something at the moment that could replace animal tests with a stroke," he said.

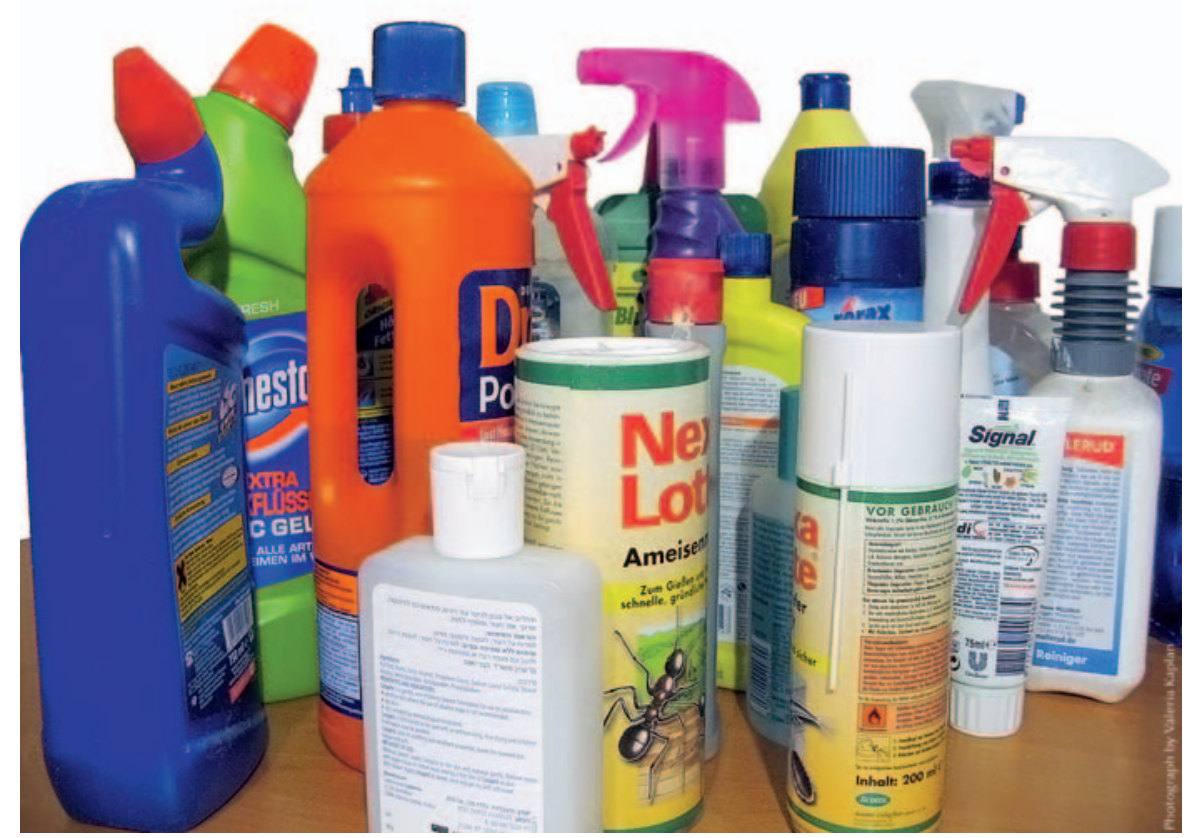

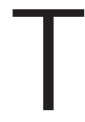
his problem is not lost on the EU, which has funded several research projects to develop, assess and validate alternative methods. "The amounts that are being invested in these programmes are quite substantial," said an EC official with the Directorate General (DG) Enterprise and Industry, who spoke on condition of anonymity. About $€ 600$ million was allocated to such projects under the EU's Sixth Framework Programme (FP6), with $€ 1$ billion planned under FP7.

\section{... the German Federal Institute for Risk Assessment... estimated that [REACH] could lead to a demand for up to 45 million laboratory animals over the next 15 years}

In vitro methods will not be able to replace all animal tests in the foreseeable future, van Ravenzwaay pointed out, because they are too limited to provide a full picture of how a substance behaves in the body. To investigate kinetic and metabolic parameters-such as how fast a compound is excreted or broken down in the liver-animal tests are necessary. "Something like a two-generation study investigates about 400 individual parameters, and there will be no simple in vitro method to replace that," van Ravenzwaay said. "It is simply not possible to reduce extremely complex biological systems to a few parameters." Furthermore, it also remains to be seen whether in vitro tests are enough to ease societies' suspicions about hazardous substances. "Nobody feels really comfortable going from an isolated cell culture to a complex organism" and extrapolating results from in vitro tests to animals or humans, Calabrese said.

"It is nearly impossible to test every single chemical," said O rtwin Renn, Professor of Sociology and an expert on risk assessment at the $U$ niversity of Stuttgart, Germany. The problem becomes even more complicated in the case of endocrine disruptors, which the EC has labelled as substances of "very high concern". The scientific evidence that these substances pose a threat to human health remains disputed (Breithaupt, 2004); as Renn pointed out, their effects are very hard to demonstrate both toxicologically and epidemiologically. "[I]t is already possible to show for a range of chemicals that they are endocrine disruptors," Santillo commented. "But there is still a long way to go to develop methods to test and identify them with high reliability." The EC is aware of this: "It's clear that the methodology has to be further developed for such tests," said the EC official.

Renn therefore thinks that the EU should shoulder some of these costs, in particular for the development and validation of alternative methods, leaving industry to pay for 
the actual testing. Santillo agrees. "Not all the burden should be put on industry," he said, adding that costs should not be a barrier to implementing REACH .

\section{... the development of invitro methods that would pass both scientific and regulatory scrutiny is a lengthy and costly undertaking}

The European Chemical Industry Council (CEFIC; Brussels, Belgium), which represents about 27,000 manufacturers and other companies, maintains that the cost of REACH will force many small and medium-size firms out of the market (CEFIC, 2003). Renn commented that some smaller companies, particularly producers of cosmetics with many different ingredients, might be disproportionately affected. "Some help [for such companies] would certainly make sense," he said, for example administrative help for registering their products. The EC estimates that in the first 11 years after its implementation, the direct costs of REACH will be $€ 3.2$ billion to the chemicals industry and $€ 2.8-3.6$ billion to downstream users.

Another EC official with DG Enterprise and Industry, who also spoke on condition of anonymity, pointed out that REACH would require industry to share its data with other producers and downstream users so as to reduce both the costs and the number of tests. In addition, companies would have to gather what is already known for a substance and perform new tests only if necessary information is missing. Unfortunately, for many existing chemicals-defined as having been introduced on the market before September 1981-data on safety and adverse effects are rare or non-existent. The current version of REACH also proposes a tiered approach, whereby the requirements for information increase with the annual production quantity of the chemical in question. This makes little sense, according to van Ravenzwaay, because "the production volume does not determine the actual exposure."

W ithout alternative methods to reduce animal tests and costs, the question then becomes how to assess 30,000 chemicals more efficiently using existing methods. Santillo and van Ravenzwaay both believe that this is possible by concentrating on those chemicals that would need more rigorous testing. By "turning the testing process on its head" van Ravenzwaay believes it would be possible to save an enormous amount of resources. Instead of a "labelling fetishism" which requires testing a chemical for all possible effects, a sensitive screening through suitable in vitro methods would be followed up with animal studies only if potential adverse effects appeared. "The available in vitro methods tend to be more sensitive than the in vivo studies. Thus, the absence of effects in these in vitro studies indicates that there will also be no effect in vivo. If we accept this and concentrate our in vivo testing on the chemicals that demonstrated an effect in vitro, we would focus our resources efficiently on those chemicals that need to be investigated," he explained.

"However, this makes [testing] much more complex. You have to think it through," van Ravenzwaay said, adding that pharmaceutical companies already use such approaches when they test drug candidates for safety. But such an approach would have other positive effects: the number of animals used to implement REACH could be reduced to fewer than 10 million if, among other factors, the current regulations demanding obligatory tests for many endpoints were replaced with more flexible strategies (H ofer et al, 2004). The latest version of REACH, on the basis of comments from the Council of Europe, includes the possibility of waiving tests if the risk assessment determines that exposure to humans or the environment is low.

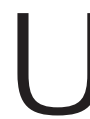

Itimately, the dispute comes down to a decision whether to ban or substitute a substance. This will be relatively straightforward for many compounds once sufficient data exist, how ever, there are still cases-most notoriously, endocrine disruptors-where neither risk nor exposure can be clearly determined. To this end, REACH explicitly cites the precautionary principle as a basis for decision making whenever the scientific data are insufficient. Renn commented that this should apply to chemicals that show a specific combination of effects: "If there is an initial suspicion [for adverse effects] and this compound is ubiquitous, persistent and bioaccumulative, then it would be precautionary to regulate it." In general, he added, one should use the precautionary principle to avoid irreversible mistakes: societies can learn from previous errors and implement rules to correct them, but this is not possible if chemicals with high ubiquity, persistence and bioaccumulation are released into the environment.

About the EC's explicit invocation of the precautionary principle, van Ravenzwaay is more cautious. "You can use the precautionary principle if there is not enough knowledge and a high exposure at the same time, but you cannot regulate everything according to the precautionary principle," he said. "It is good that compounds will be thoroughly and sensibly tested, but the [goal] should be risk assessment and not going directly to risk management."

Santillo, by contrast, welcomes the precautionary principle. "W hat we can hope for is that the science of toxicology is much more used to making precautionary predictions," he said. "It has to provide ways to allow decision making but in a way in which wrong decisions have less serious implications." Santillo stressed that Greenpeace is not calling for a ban per se on all chemicals with negative effects. "What we're not saying and what the European Parliament is not saying, is that we need an immediate ban ... if the use is essential to society and if no alternative exists." For him, it is more a matter of keeping human and environmental health in mind. "The difficulty that we see is in regard to where the threshold is set," Santillo commented. "In the end, it comes down to how much trust is one prepared to put on the threshold, and who is given the benefit of the doubt: consumers or producers."

\section{... societies can learn from previous errors and implement rules to correct them, but this is not possible if chemicals with high ubiquity, persistence and bioaccumulation are released into the environment}

Notwithstanding the disputes over details, there is agreement among all quarters that REACH is a much-needed legislation. Even industry, while critical about its implementation, largely welcomes it. "There is a need for a manageable and enforceable legislation that is able to be applied both by industry and by the authorities, and which is able to fulfil 
the expectations of society," CEFIC stated (2003). "REACH has its good sides," van Ravenzwaay said, because it could become a starting point for more research in toxicology and an improved risk assessment. Moreover, REACH would finally shed light on the health and environmental risks of many chemicals that have been in use for decades but were never assessed by safety tests that are now mandatory. In doing so, it will finally end an almost century-long experiment on the human race, in which regulatory agencies have abstained from requiring sufficient safety data for a large number of chemicals. "O ne can consider the ethics of throwing 100,000 chemicals on humans without having them tested," Calabrese said. "We're using human society as a guinea pig."

\section{REFEREN CES}

Breithaupt H (2004) A cause without a disease. EMBO Rep 5: 16-18

CEFIC (2003) Consultation D ocument concerning

Registration, Evaluation, Authorisation and

Restriction of Chemicals (REACH) Executive

Summary. Brussels, Belgium: European Chemical Industry Council
EC (2003) Proposal for a Regulation of the European Parliament and of the Council concerning the Registration, Evaluation, Authorisation and Restriction of Chemicals (REACH), establishing a European Chemicals Agency and amending Directive 1999/45/EC and Regulation (EC). COM (03) 644(01). Brussels, Belgium: European Commission

EEC (1967) Council Directive 67/548/EEC of 27 June 1967 on the approximation of laws, regulations and administrative provisions relating to the classification, packaging and labelling of dangerous substances. Brussels, Belgium: European Commission

EEC (1986) Council Directive of 24 N ovember 1986 on the approximation of laws, regulations and administrative provisions of the M ember States regarding the protection of animals used for experimental and other scientific purposes (86/609/EEC). Brussels, Belgium: European Commission

H oferT, Gerner I, G undert-Remy U, Liebsch M, Schulte A, Spielmann H, Vogel R, Wettig K (2004) Animal testing and alternative approaches for the human health risk assessment under the proposed new European chemicals regulation. Arch Toxicol 78: 549-564

Matthiessen L, Lucaroni B, Sachez E (2003)

Towards responsible animal research. EM BO Rep 4: 104-107

\section{HolgerBreithaupt}

doi:10.1038/sj.embor.7400816

\title{
A quantum leap in biology
}

\author{
One inscrutable field hel ps another, as quantum physics unravels \\ consciousness
}

$\mathrm{T}$ he most esoteric research field in the natural sciences is probably quantum physics. Despite the fact that Werner Heisenberg first proposed its central concepts nearly 80 years ago, it continues to baffle physicists and to cause headaches among non-physicists. Even Albert Einstein was unwilling to accept the central tenet that everything is just a matter of possibilities; he famously dismissed Heisenberg's ideas by asserting that "God does not throw dice." As quantum physics seems too mystical to be relevant to anything as real as a living organism, it might come as a surprise that its first applications have arrived in biology, rather than physics.

The seeds of contemporary quantum biology were sown as early as 1930, a mere three years after Heisenberg postulated his uncertainty principle describing the inability to measure related quantities exactly (see sidebar). At that time, Erich Hückel, a German chemist and physicist, developed simplified methods based on quantum mechanics (Q M ) for analysing the structure of unsaturated organic molecules, in particular to explain the state of electrons in aromatic compounds. But Hückel was too far ahead of his time, and his concepts w ent almost completely unrecognized until the 1950s, when the arrival of computers made it possible to perform more detailed calculations. It was not until the 1990s, however, that the field of quantum biology became established with the development of density functional theory (DFT), which allows accurate calculations of electronic structure (see sidebar). By that time, high-resolution structures of protein complexes obtained using X-ray crystallography and nuclear magnetic resonance produced sufficiently accurate descriptions of crucial molecules for QM methods to unravel the details of key reactions, such as ATP hydrolysis.

QM has also made a significant impact on the study of photoreception and the detection of colour, on research into the sensing of magnetic location and directional information by migratory birds, and, most controversially, in understanding the processes underlying consciousness. The last example relies on certain unprovable assumptions about the scientific basis of perception, whereas research on catalytic reaction centres (such as analysing substrate binding) hinges on solving the Schrödinger wave equation. Described in 1926, and central to the theory of $\mathrm{QM}$, this equation describes the probability that a given electron is in a particular location at a certain time (see sidebar). Such Q M-based applications calculate the sequence of events at the atomic level by analysing the electronic properties during the formation and breakage of chemical bonds or the orientation of electron orbitals, as determined by their quantum wave function.

$\mathrm{T}$ he validity of Q M methods is not seriously disputed, but their high computational intensity precluded their use until the 1990s. Although computers had been used to simulate the function of proteins and their chemical reactions since the mid1960s, these calculations were based on molecular mechanics (MM) techniques derived from N ewton's laws of motion. These operate at the level of molecules rather than electrons, and describe the energy and forces associated with particular protein structures, by studying simpler model compounds that mimic the chemical groups in the constituent amino acids and other components.

\section{The seeds of contemporary quantum biology were sown as early as 1930, a mere three years after H eisenberg postulated his uncertainty principle...}

The weakness of M M methods is that they rely on making simple assumptions. For example, electrons are not considered directly, but are assumed to be in an optimum position determined by the location of their atomic nuclei. This process, based on the Born-Oppenheimer approximation of 